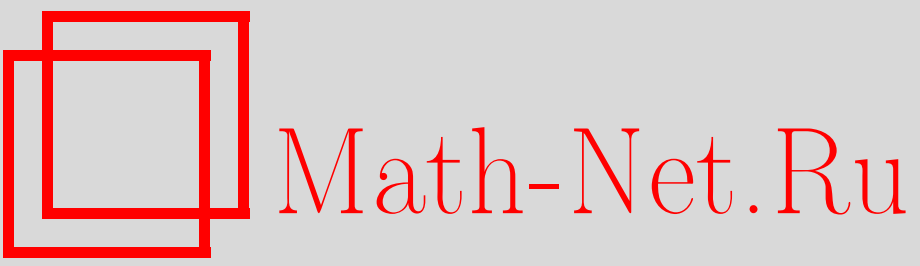

Е. Ю. Юденкова, Бесконечная линейная независимость значений обобщенных гипергеометрических рядов с иррациональными параметрами в полиадических точках, Итоги науки и техн. Сер. Соврем. мат. и ее прил. Темат. обз., 2020, том $179,88-93$

DOI: https://doi.org/10.36535/0233-6723-2020-179-88-93

Использование Общероссийского математического портала Math-Net.Ru подразумевает, что вы прочитали и согласны с пользовательским соглашением

http://www.mathnet.ru/rus/agreement

Параметры загрузки:

IP : 34.229 .108 .108

26 апреля 2023 г., 16:49:29 


\title{
БЕСКОНЕЧНАЯ ЛИНЕЙНАЯ НЕЗАВИСИМОСТЬ ЗНАЧЕНИЙ ОБОБЩЕННЫХ ГИПЕРГЕОМЕТРИЧЕСКИХ РЯДОВ С ИРРАЦИОНАЛЬНЫМИ ПАРАМЕТРАМИ В ПОЛИАДИЧЕСКИХ ТОЧКАХ
}

\author{
(c) 2020 г. $\quad$ Е. Ю. ЮДЕНКОВА
}

\begin{abstract}
АннотАция. Работа посвящена доказательству бесконечной линейной независимости в точках, допускающих высокие порядки приближения алгебраическими числами в неархимедовски нормированных полях.
\end{abstract}

Ключевые слова: гипергеометрический ряд, иррациональный параметр.

\section{INFINITE LINEAR INDEPENDENCE}

OF VALUES OF GENERALIZED HYPERGEOMETRIC SERIES WITH IRRATIONAL PARAMETERS AT POLYADIC POINTS

\author{
(c) 2020 E. YU. YUDENKOVA
}

Abstract. The paper is devoted to the proof of infinite linear independence at points that admit high-order approximations by algebraic numbers in non-Archimedean normalized fields.

Keywords and phrases: hypergeometric series, irrational parameter.

AMS Subject Classification: $33 \mathrm{C} 20$

Обобщенные гипергеометрические ряды с рациональными параметрами, в зависимости от их вида, входят либо в классы $E$-функций или $G$-функций, либо в класс $F$-рядов. Арифметическим свойствам $E$-функций посвящены работы $[9,11]$. В них приведена обширная библиография. Арифметические свойства $F$-рядов описаны в $[2-8,10]$.

Введем обозначение

$$
F\left(\mu_{1}, \ldots, \mu_{m}, z\right)=\sum_{n=0}^{\infty} \frac{\left(\mu_{1}\right)_{n} \ldots\left(\mu_{m}\right)_{n}}{n !} z^{n}
$$

где

$$
(\mu)_{0}=1, \quad(\mu)_{n}=\mu(\mu+1) \ldots(\mu+n-1), \quad n \geqslant 1,
$$

- символ Похгаммера. Положим

$$
\begin{aligned}
f_{0}(z) & =F\left(\alpha_{1}, \alpha_{2}, \ldots, \alpha_{m}, z\right), \\
f_{1}(z) & =F\left(\alpha_{1}+1, \alpha_{2}, \ldots, \alpha_{m}, z\right), \\
& \ldots \\
f_{m-1}(z) & =F\left(\alpha_{1}+1, \alpha_{2}+1, \ldots, \alpha_{m-1}+1, \alpha_{m}, z\right) .
\end{aligned}
$$


Считается, что $\alpha_{1}, \ldots, \alpha_{m-2}$ - целые числа из поля $\mathbb{K}$, отличные от 0 и отрицательных целых чисел. Пусть $\alpha_{m-1}=\alpha_{m}=1$.

Для любого нормирования $v$ поля $\mathbb{K}$ и любого целого числа $z$ из поля $\mathbb{K}$ все ряды $(2)$ - целые числа из поля $\mathbb{K}$.

В [4] с использованием аппроксимаций Эрмита-Паде из [1] установлена следующая теорема.

Теорема 1. Пусть $\alpha_{1}, \ldots, \alpha_{m-2}, h_{0}, \ldots, h_{m-1} \in \mathbb{Z}_{\mathbb{K}}$ и среди чисел $h_{0}, \ldots, h_{m-1}$ есть отличные от 0 , где $\mathbb{Z}_{\mathbb{K}}-$ кольияо чельх чисел поля $\mathbb{K}$.

Тогда существует бесконечное множество нормирований $v$ поля $\mathbb{K}$ таких, что в кольце $\mathbb{K}_{v}$

$$
\left|h_{0} f_{0}(\xi)+\ldots+h_{m-1} f_{m-1}(\xi)\right|_{v} \neq 0 .
$$

Цель настоящей работы - доказать аналогичную теорему для точки $\xi$, хорошо приближаемой целыми числами из $\mathbb{K}$.

Пусть

$$
\xi=\sum_{r=0}^{\infty} \theta_{r},
$$

$\theta_{r} \in \mathbb{Z}_{\mathbb{K}}$, причем пусть этот ряд сходится во всех $\mathbb{K}_{v}, v \in V_{0}$ ( $V_{0}$ обозначает множество всех неархимедовых нормирований поля $\mathbb{K}$ ). Пусть $\epsilon>0$ и пусть существует бесконечное множество $A$ таких целых чисел $a$ из поля $\mathbb{K}$, что для всех простых чисел $p$, удовлетворяющих условию

$$
p \leqslant m \exp \left(\ln ^{(1+\epsilon)}|\bar{a}|\right)
$$

(здесь $|\bar{a}|$ обозначает максимум модулей сопряженных с $a \in \mathbb{K}$ чисел) и любого нормирования $v$, продолжающего $p$-адическое нормирование, при некотором $\delta>0$ выполняется неравенство

$$
|\xi-a|_{v}<\exp \left(-(m-1+\delta) \exp \left(\ln ^{(1+\epsilon)}|\bar{a}|\right) \ln ^{(1+\epsilon)}|\bar{a}|\right) .
$$

Теорема 2. Пусть числа $\alpha_{1}, \ldots, \alpha_{m-2}, h_{0}, \ldots, h_{m-1} \in \mathbb{Z}_{\mathbb{K}}$ u среди чисел $h_{0}, \ldots, h_{m-1}$ есть отличные от 0. Пусть точки $\xi$ вида (4) удовлетворяют условиям (5), (6). Тогда существует бесконечное множество таких нормирований $v$ поля $\mathbb{K}$, что в поле $\mathbb{K}_{v}$ выполняется неравенство (3).

Доказательство. В доказательстве теоремы используются леммы из [4].

Пусть $c_{i}$ - положительные постоянные, $S_{i}$ - натуральные числа. Пусть $N$ - натуральное число. Определим числа $r, s, t, l$ условиями

$$
N=m s+r=(m-1) t+l, \quad 1 \leqslant r \leqslant m, 1 \leqslant l \leqslant m-1 ;
$$

при этом

Положим

$$
s=\left[\frac{N-1}{m}\right], \quad t=\left[\frac{N-1}{m-1}\right] .
$$

$$
\begin{aligned}
\alpha_{N} & =\alpha_{r}+s, \\
f_{N}(z) & =F\left(\alpha_{N+1}, \ldots, \alpha_{N+m}, z\right), \\
u_{N}(z) & =\alpha_{1} \ldots \alpha_{N} z^{t} f_{N}(z) .
\end{aligned}
$$

Лемма 1. При любом натуральном $N$ существуют такие многочлены $P_{N, 0}(z), \ldots, P_{N, m-1}(z)$, чmo

$$
u_{N}(z)=P_{N, 0}(z) u_{0}(z)+\ldots+P_{N, m-1}(z) u_{m-1}(z) .
$$

При этом степени многочленов $P_{N, 0}(z), \ldots, P_{N, m-1}(z)$ не превосходлт числа $t-s$, рлдъ (11) линейно независимы над $\mathbb{K}(z)$, и выполняются рекуррентные соотношения

$$
u_{N+m}(z)=u_{N+1}(z)-\alpha_{N+1} u_{N}(z),
$$

если $N=0$ или $N \geqslant 1$ и $N$ не делится на $m-1$,

$$
u_{N+m}(z)=u_{N+1}(z)-\alpha_{N+1} z u_{N}(z),
$$


если $N \geqslant 1$ и $N$ делится на $m-1$, и для любого $i=0,1, \ldots, m-1$

$$
P_{N+m, i}(z)=P_{N+1, i}(z)-\alpha_{N+1} P_{N, i}(z),
$$

если $N=0$ или $N \geqslant 1$ и $N$ не делится на $m-1$,

$$
P_{N+m, i}(z)=P_{N+1, i}(z)-\alpha_{N+1} z P_{N, i}(z),
$$

если $N \geqslant 1$ и $N$ делится на $m-1$. При этом для всех неотричательных целых значений $N$ имеет место равенство

$$
\Delta_{N}(z)=(-1)^{m N} \alpha_{1} \ldots \alpha_{N} z^{t}
$$

где

$$
\Delta_{N}(z)=\left|P_{N+j, i}\right|_{i, j=0, \ldots, m-1} .
$$

Для дальнейшего будет полезным понятие размера многочлена $P(z)$ с коэффициентами из поля $\mathbb{K}$. Эта величина определяется как наибольший из модулей всех чисел, сопряженных с коэффициентами многочлена в поле $\mathbb{K}$, и обозначается $|\overline{P(z)}|$. Установим простые свойства размера многочлена:

и для $\alpha \in \mathbb{K}$

$$
\left|\overline{P_{1}(z)+P_{2}(z)}\right| \leqslant\left|\overline{P_{1}(z)}\right|\left|\overline{P_{2}(z)}\right|
$$

$$
|\overline{\alpha P(z)}| \leqslant|\bar{\alpha}||\overline{P(z)}| .
$$

Неравенства (19) и (20) сразу следуют из того факта, что для чисел $\gamma, \delta$ из поля $\mathbb{K}$ любое число, сопряженное с суммой этих чисел, является суммой сопряженных с ними чисел, а любое число, сопряженное с произведением этих чисел, является произведением сопряженных с ними чисел.

Введем обозначение

$$
c_{0}=\left[\max \left(\left|\overline{\alpha_{1}}\right|, \ldots,\left|\overline{\alpha_{m}}\right|\right)\right]+1 .
$$

Поскольку $\alpha_{m-1}=1$, то $c_{0} \geqslant 2$.

Лемма 2. В условиях теоремы для $N=m s+r, 1 \leqslant r \leqslant m, u$ для любого $t=0, \ldots, q$ имеет место неравенство

$$
\left|\overline{P_{N, 1}(z)}\right| \leqslant c_{0}^{N}\left(c_{0}+1\right) \ldots\left(c_{0}+s\right) .
$$

Продолжим доказательство теоремы. В любом поле $\mathbb{K}$ рассмотрим элемент этого поля, имеющий вид

$$
L(\xi)=h_{1} f_{0}(\xi)+\ldots+h_{m} f_{m-1}(\xi) .
$$

Умножим его на $\alpha_{1} \ldots \alpha_{m-1}$ и получим (с учетом равенств (11)) элемент

$$
L *=b_{0} u_{0}(\xi)+\ldots+b_{m-1} u_{m-1}(\xi),
$$

представляющий собой линейную форму от $u_{0}(\xi), \ldots, u_{m-1}(\xi)$ с коэффициентами, являющимися целыми числами из поля $\mathbb{K}$. Поскольку $L(\xi) \neq 0$, полученная форма $(24)$ также является ненулевой. Обозначим

$$
H=\max _{i=0, \ldots, m-1}\left|\overline{b_{1}}\right| .
$$

Рассмотрим при некотором натуральном $N$ линейные формы

$$
u_{N}(\xi), \ldots, u_{N+m-1}(\xi)
$$

от $u_{0}(\xi), \ldots, u_{m-1}(\xi)$, определенные в $(12)$. Определитель матрицы из коэффициентов $\Delta_{N}(\xi)$ отличен от нуля ввиду равенства (17) и условий теоремы. Поэтому среди форм (26) существуют $m-1$ форм, линейно независимых с формой $L *$. Следовательно, определитель этой совокупности форм

где

$$
\Delta_{N}^{*}(\xi)=\left|\begin{array}{ccc}
b_{0} & \ldots & b_{m-1} \\
P_{N_{1}, 0}(\xi) & \ldots & P_{N_{1}, m-1}(\xi) \\
\cdots & \ldots & \ldots \\
P_{N_{m-1}, 0}(\xi) & \ldots & P_{N_{m-1}, m-1}(\xi)
\end{array}\right|,
$$

$$
N_{i} \in\{N, \ldots, N+m-1\}
$$


отличен от нуля, т.е. $\Delta_{N}^{*}(\xi) \neq 0$. Величину $(27)$ можно рассматривать как значение многочлена $\Delta_{N}^{*}(z)$ в точке $z=\xi$.

Лемма 3. При $s \geqslant S_{0}$ имеет место неравенство

$$
\prod_{v \in V_{0}}\left|\Delta_{N}^{*}(z)\right| \geqslant H^{-1} e^{-(m-1) s \ln s-c_{1} s},
$$

где произведение в левой части взято по всем неархимедовым нормированиям поля $\mathbb{K}$.

Доказательство. Именно в этой лемме требуются изменения в рассуждениях из [4]. Доказательство, до определенного момента, совпадает с доказательством леммы 3 из [4]. Из равенства (27) следует, что $\Delta_{N}^{*}(\xi)$ - многочлен в коэффициентами, являющимися целыми числами из поля $\mathbb{K}$. Кроме того, ввиду (28) имеем $N_{i} \leqslant N+m-1$. Пусть $\tilde{s}$ и $\tilde{t}$ определены равенствами

$$
N+m-1=m \tilde{s}+\tilde{r}=(m-1) \tilde{t}+\tilde{l} .
$$

При этом

$$
\tilde{s}=\left[\frac{N+m-2}{m}\right], \quad \tilde{t}=\left[\frac{N+m-2}{m-1}\right] .
$$

Из соотношений (9) и (30) следует, что $s \leqslant \tilde{s} \leqslant s+1$, а $\tilde{t}=t+1$. Поэтому, используя неравенство (22), с заменой $s$ на $s+1$ получаем

$$
\left|\overline{P_{N_{i}, j}(z)}\right| \leqslant c_{0}^{N+m-1}\left(c_{0}+1\right) \ldots\left(c_{0}+s+1\right), \quad j=0, \ldots, m-1,
$$

откуда с учетом $(25)$ имеем

$$
\left|\overline{\Delta_{N}^{*}(z)}\right| \leqslant m ! H c_{0}^{(m-1)(N+m-1)}\left(\left(c_{0}+1\right) \ldots\left(c_{0}+s\right)\right)^{m-1} .
$$

Представим произведение $\left(c_{0}+1\right) \ldots\left(c_{0}+s+1\right)$ в виде

$$
\left(c_{0}+1\right) \ldots\left(c_{0}+s+1\right)=\frac{\Gamma\left(c_{0}+s+2\right)}{\Gamma\left(c_{0}+1\right)},
$$

где $Г(x)$ - гамма-функция Эйлера.

Лемма 4. Для любой постоянной величины а и любого $\delta>0$ при $|s| \rightarrow \infty$ равномерно при $-\pi+\delta \leqslant \arg s \leqslant \pi-\delta$ имеет место равенство

$$
\ln \Gamma(s+a)=\left(s+a-\frac{1}{2}\right) \ln s-s+\frac{1}{2} \ln 2 \pi+O\left(\frac{1}{|s|}\right) .
$$

Из (32) и (33) сразу следует, что при $s \rightarrow \infty$

$$
\left(c_{0}+1\right) \ldots\left(c_{0}+s+1\right)=\exp (s \ln s+O(s)) .
$$

Из неравенства (31) и равенства (34) вытекает, что при $s>S_{1}$ с некоторой постоянной $c_{2}$

$$
\left|\overline{\Delta_{N}^{*}(z)}\right| \leqslant H \exp \left((m-1) s \ln s+c_{2} s\right) .
$$

Далее, согласно (13) степень многочлена $\Delta_{N}^{*}(z)$ не превосходит числа

$$
\begin{aligned}
(m-1)(\tilde{t}-\tilde{s}) \leqslant(m-1) & \left(\left[\frac{N+m-2}{m-1}\right]-\left[\frac{N-1}{m}\right]\right) \\
& \leqslant(m-1)\left(\frac{N+m-2}{m-1}-\frac{N-1}{m}+1\right) \leqslant \frac{N}{m}+2(m-1) \leqslant s+2(m-1) .
\end{aligned}
$$

Это означает, что для любого $a \in \mathbb{Z}_{\mathbb{K}}$

$$
\left|\overline{\Delta_{N}^{*}(a)}\right| \leqslant H \exp \left((m-1) s \ln s+c_{2} s\right)|\bar{a}|^{s+2(m-1)}
$$

согласно (36).

Следовательно, для любого $v$

$$
\left|\Delta_{N}^{*}(a)\right|_{v} \geqslant \frac{1}{\left|\overline{\Delta_{N}^{*}(a)}\right|} \geqslant H^{-1} \exp \left(-\left((m-1) s \ln s+c_{2} s\right)\right)|\bar{a}|^{-s-2(m-1)} .
$$


Докажем, что для достаточно большого $S$ выполняется равенство

$$
\left|\Delta_{N}^{*}(a)\right|_{v}=\left|\Delta_{N}^{*}(\xi)\right|_{v}
$$

Для этого заметим, что

$$
\Delta_{N}^{*}(\xi)=\Delta_{N}^{*}(a)+(\xi-a) \Delta_{0}(\xi, a)
$$

где $\Delta_{0}(\xi, a)$ - многочлен от $\xi$ и $a$ с целыми коэффициентами из поля $\mathbb{K}$.

Во всех рассматриваемых полях $\mathbb{K}$ имеем

$$
|\xi-a|_{v}\left|\Delta_{0}(\xi, a)\right|_{v}=|\xi-a|_{v} \leqslant\left|(\xi-a) \Delta_{0}(\xi, a)\right|_{v}<\exp \left(-(m-1+\delta) \exp \left(\ln ^{1+\epsilon}|\bar{a}|\right)\right) \ln ^{1+\epsilon}|\bar{a}| .
$$

Выберем параметры $s$ и $a$ так, чтобы оценки сверху для $|\xi-a|_{v}$ стала меньше, чем оценка сверху для $\left|\Delta_{n}^{*}(a)\right|_{v}$. Тогда из равенства (37) будет следовать, что

$$
\left|\Delta_{N}^{*}(\xi)\right|_{v}=\left|\Delta_{N}^{*}(a)\right|_{v}
$$

Для этого положим $s=\left[\exp \left(\ln ^{1+\epsilon}|\bar{a}|\right)\right]+1$; тогда

$$
\ln ^{1+\epsilon}|\bar{a}| \leqslant \ln s \leqslant \ln ^{1+\epsilon}|\bar{a}|+1 .
$$

Докажем, что при указанном выборе $s$ при $s \geqslant S_{0}$ (что означает, что выбрано достаточно большое значение $|\bar{a}|)$

$$
\begin{aligned}
\left|\Delta_{N}^{*}(a)\right|_{v} \geqslant H^{-1} \exp \left(-(m-1) s \ln s+c_{2} s\right)|\bar{a}|^{-s-2(m-1)}> & \\
& >\exp \left(-(m-1+\delta) \exp \left(\ln ^{(1+\epsilon)}|\bar{a}|\right) \ln ^{(1+\epsilon)}|\bar{a}|\right)>|\xi-a|_{v} .
\end{aligned}
$$

Эти неравенства будут следовать из неравенства

$$
\ln H+(m-1) s \ln s-c_{2} s+(s+2(m-1)) \ln |\bar{a}|<(m-1+\delta) \exp \left(\ln ^{1+\epsilon}|\bar{a}|\right) \ln ^{1+\epsilon}|\bar{a}| .
$$

С учетом соотношений (38) последнее неравенство вытекает из неравенства

$$
\ln H+c_{2} s+(s+2(m-1)) \ln ^{s /(1+\epsilon)}<\delta s \ln s,
$$

справедливого при $s \geqslant S_{0}$. Поэтому из (35) и (36) при $s>S_{2}$ с некоторой постоянной $c_{3}$ получаем неравенство

Поскольку

$$
\left|\overline{\Delta_{N}^{*}(\xi)}\right| \leqslant H \exp \left((m-1) s \ln s+c_{3} s\right) .
$$

$$
\prod_{v \in V_{\infty}}\left|\overline{\Delta_{N}^{*}(\xi)}\right|_{v} \leqslant \prod_{v \in V_{\infty}}\left|\overline{\Delta_{N}^{*}(\xi)}\right|^{\kappa_{v} / \kappa}=\left|\overline{\Delta_{N}^{*}(\xi)}\right|
$$

из неравенства (39) следует неравенство (29) с соответствующими $S_{0}, c_{1}$. Лемма доказана.

Лемма 5. Пусть $v \in V_{0},|\xi|_{v} \leqslant 1$. Тогда существует такое число $i, i \in\{0, \ldots, m-1\}$, что $\left|f_{i}(\xi)\right|_{v}=1 u\left|u_{i}(\xi)\right|_{v}=1$.

Дальнейшее доказательство дословно повторяет доказательство теоремы из [4] и поэтому опущено.

\section{СПИСОК ЛИТЕРАТУРЫ}

1. Нестеренко Ю. В. Приближения Эрмита-Паде обобщенных гипергеометрических функций// Мат. сб. $-1994 .-185$, № 3. - С. 39-72.

2. Чирский В. Г. О глобальных соотношениях// Мат. заметки. - 1990. - 48, № 2. - С. 123-127.

3. Чирский В. Г. Об алгебраических соотношениях в неархимедовски нормированных полях// Функц. анал. прилож. -1992 . -26 , № 2. - С. 41-50.

4. Чирский В. Г. Об арифметических свойствах обобщенных гипергеометрических рядов с иррациональными параметрами// Изв. РАН. Сер. мат. - 2014. - 78, № 6. - С. 193-210.

5. Чирский В. Г. Арифметические свойства полиадических рядов с периодическими коэффициентами// Докл. РАН. - 2014. - 459, № 6. - С. 677-679.

6. Чирский В. Г. Об арифметических свойствах ряда Эйлера// Вестн. Мос. Ун-та. Сер. 1. Мат. Мех. 2015. - № 1. - C. 59-61. 
7. Чирский В. Г. Арифметические свойства полиадических рядов с периодическими коэффициентами// Изв. РАН. Сер. мат. - 2017. - 81, № 2. - С. 215-232.

8. Чирский В. Г. Арифметические свойства обобщенных гипергеометрических $F$-рядов// Докл. РАН. 2018. - 483, № 3. - С. $252-254$.

9. Шидловский А. Б. Трансцендентные числа. - М.: Наука, 1987.

10. Bertrand D., Chiskii V., Yebbou J. Effective estimates for global relations on Euler-type series// Ann. Fac. Sci. Toulouse. - 2004. - 13, № 2. - P. 241-260.

11. Chirskii V. G. Topical problems of the theory of transcendental numbers: Developments of approaches to tyeir solutions in works of Yu. V. Nesterenko// Russ. J. Math. Phys. — 2017. — 24, № 2. — P. $153-171$.

Юденкова Екатерина Юрьевна

Московский педагогический государственный университет

E-mail: yudenkovaey@gmail.com 